\title{
DESIGN AND CONTROL OF A STEERING WHEEL VIBRATION SIMULATOR
}

\author{
James A. Mynderse and George T.-C. Chiu \\ Perception Based Engineering Center \\ Ray W. Herrick Laboratories \\ Purdue University \\ West Lafayette, Indiana 47907, USA \\ E-mail: mynderse@purdue.edu \\ E-mail: gchiu@purdue.edu
}

\begin{abstract}
The design and control of a steering wheel vibration simulator capable of reproducing a set of desired vibration/acceleration signals is presented. The simulator is to be used in characterizing human perception of vibration as transmitted to the hand through the steering wheel. Accelerometers were used to record the acceleration at the top of the steering wheel in both the up-down (z) and side-to-side (y) directions. A two degree-of-freedom controller was synthesized with a stabilizing feedback controller designed using linear matrix inequality techniques and a zero phase error tracking feedforward controller. Simulation and experimental results to verify key steps of the design process and the effectiveness of the simulator are presented. Copyright $@ 2005$ IFAC
\end{abstract}

Keywords: automatic control, closed-loop controllers, stabilizing feedback, feedforward compensation, vibration.

\section{INTRODUCTION}

In order to design a product that will be accepted by the intended consumer, the product must be perceived as being of the appropriate quality. In the automobile industry, the consumer has certain preconceived expectations of the fuel efficiency, handling, ride comfort and other quality factors that must be met in order to facilitate a purchase of the product. One component in the perception of quality has been found to be the vibrations transmitted from the seat, floor pan and steering wheel to the driver. Many auto makers, e.g. Ford Motor Company, as well as research institutions have developed facilities to generate controlled vibrations for testing human vibration perception. These facilities typically consist of multiple degrees-of-freedom actuators under a car seat and under the replica of the front section of the vehicle. The floor pan and seat vibrations produced by these facilities have been sufficient for threshold testing. However, there are only a limited number of devices for reproducing steering wheel vibration. Most of them involve fitting a production steering wheel and steering wheel column to the existing vibration simulator. When using these facilities it is often difficult to generate repeatable stimuli under various different operating conditions. To study human perception of vibration it is extremely important to be able to generate repeatable, high quality stimuli. Therefore, it is the objective of this study to develop a steering wheel vibration simulator that can accurately generated the desired vibration stimuli under a wide range of operating conditions.

Many researchers have made progress that aided this project. Bolanowski, et al. (1988) identified the four nerve fibers and four channels involved in tactile sensation. Gescheider, et al. (1983) proved the independence of the channels. Brisben, et al. (1999) tested the detection thresholds for the palm in two axes. In control design, Balakrishnan, et al. (1994), detailed LMI controller design for continuous systems and both Iwasaki (1993) and Skelton, et al. (1998) showed the discrete formulation of the LMI design process. This 
work involved the gripping of a steering wheel instead of the hand tool typically used in haptics studies. The steering wheel introduces vibration in a combination of the traditional axes used. The controller was designed in the discrete domain.

The remainder of the paper details the design, modeling and control of a steering wheel vibration simulator which is organized as follows. Section 2 describes the design and construction of the simulator apparatus. Included are design specifications and the hardware selected. Section 3 explains the modeling procedure used to develop the plant model used in designing the controller. Section 4 outlines the procedure used in the development of the two degreeof-freedom controller. Section 5 presents simulation and experimental results of the controller and plant model as well as the controller and simulator. Summary and conclusions are given in Section 6.

\section{STEERING WHEEL VIBRATION SIMULATION APPARATUS}

\subsection{Design Specifications}

A rigid structure was built to support the steering wheel in a configuration such that the axes of all actuators were parallel to each other and to the axis of the steering wheel which they were intended to actuate. The base of the structure was mounted to a large thermodynamic shaker which was isolated from the ground and an inertial force actuator was mounted to an aluminum shaft representing the steering column in an automobile which was, in turn, connected to the steering wheel, see Fig. 1. The coordinate system used with the simulator was defined such that the z- and yaxes were in the plane of the wheel with the $\mathrm{z}$-axis in the up-down direction and the y-axis in the side-to-side direction. The x-axis extended toward the operator perpendicular to the plane defined by the wheel.

Design specifications were given as follows:

- Frequency Range: 1-250 Hz.

- Degrees-of-Freedom: Vertical.

- Static Load Support: weight of hand and arms.

- Background vibration experienced when gripping the wheel should be imperceptible.

- Simulator dimensions is based on a 2000 Ford Taurus

\subsection{Simulator Assembly}

The assembly of the simulator apparatus was comprised of three key components. These components were: the base and support column, the steering column and steering wheel and the actuator and accelerometers. Each component will be briefly discussed.

In order to minimize the effect of background vibration, it was desired to isolate the simulator from the ground. A thermodynamic shaker of approximately 3000-lbs was used as the base of the apparatus. The thermodynamic shaker included a ground vibration isolation system and convenient mounting options. A support column was necessary to elevate the steering column to the correct height relative to the ground in order to match the dimensions of a 2000 Ford Taurus.

A steering column was needed to position the steering wheel away from the thermodynamic shaker. The flexibility of both the steering column and the steering wheel added to the complexity of the plant model. In addition, the steering wheel introduced coupling between the z- and y-axes. The base was sufficiently heavy that the steering column and wheel could be modeled as a cantilever beam.

Two accelerometers were mounted to the steering wheel at the 12'o'clock position to measure acceleration in the $z$ - and $y$-axes. A single inertial force actuator was mounted parallel to the $\mathrm{z}$-axis on the steering column behind the steering wheel. Only the zaxis motion was to be controlled in this work, making the plant a one-input two-output system. The placement of the accelerometers and actuator can be seen in Fig. 1.

\subsection{Controller Hardware}

The three pieces of controller hardware used in this study were a Windows-based PC with MATLAB, a real-time feedback controller and an amplifier for the inertial force actuator.

The PC was necessary to design the controller and interface with the feedback controller. Additionally, the feedforward controller was implemented by preprocessing the reference signals. For this project, MATLAB was used for the controller design and a DSP based dynamic signal analyzer (SigLab) was used to generate the reference input from MATLAB data files.

A dSPACE ACE1104 controller rapid prototyping kit was used to implement the feedback controller. Three ADC channels were for the two accelerometers and the reference input. One DAC channel was needed to connect the feedback controller output to the actuator amplifier. Based on the frequency range specification, a minimum sample rate of $2.5-\mathrm{kHz}$ was selected. Since the low frequency vibrations from $1-$ to $20-\mathrm{Hz}$ is to be generated by the thermodynamic shaker, a $70-\mathrm{W}$ two channel audio amplifier with frequency range from 20$\mathrm{Hz}$ to $1-\mathrm{kHz}$ was used to power the inertial force actuator.

\section{SYSTEM MODELING}

After the apparatus had been assembled, a plant model 
of the system needed to be developed using the inertial force actuator as input and the accelerometer measurements as output. This was accomplished by identifying a plant model from a stepped sinusoidal response of the simulator.

The stepped sine wave input was generated by the SigLab unit and sent to the amplifier via a direct feed. Sinusoidal inputs were used for frequencies between 5$\mathrm{Hz}$ and $250-\mathrm{Hz}$ at a resolution of $1-\mathrm{Hz}$ and between $250-\mathrm{Hz}$ and $500-\mathrm{Hz}$ at a resolution of 5-Hz. All inputs were of magnitude 0.25 -volts. A 10 -msec delay was given between the start of the sinusoidal input and the data collection. For each frequency, input and output data was collected three times and the resulting frequency responses averaged.

Because the data available was frequency-based, the MATLAB fitsys command was used to generate a plant model from the frequency response data. Models were generated between $20^{\text {th }}$ and $60^{\text {th }}$ order and the sum of the square of the error between the measured frequency response plot and the frequency response of the generated system model were compared. The model with the lowest sum of squared error was determined to be the closest fit. Due to computational problems associated with high order models, it was necessary to select a model with both low error and low model order. Techniques such as the AIC (Akaike, 1972) or MAICE (Akaike, 1974) can be used to select the model order, but a heuristic approach was used instead. The best model was a $24^{\text {th }}$ order model. It is evident that the fitsys command did an excellent job fitting the $\mathrm{z}$-axis while fitting only the highest peaks of the y-axis. Figure 2 shows the experimental frequency response of the simulator and the $24^{\text {th }}$ order model, where the dotted line represents the simulator response and the solid line represents the model response.

\section{CONTROLLER SYNTHESIS}

\subsection{Stabilizing Feedback Controller}

One of the key issues for a steering wheel simulator was a lack of methods with which to force the operator to grip the steering wheel in exactly the same manner for each test. Particular areas of the steering wheel were marked for operator to place his hands, force sensors could be utilized to maintain constant grip strength, but it can not be guaranteed that each operator will grip the wheel in exactly the same fashion for each test. This variation in grip changed the system frequency response, which was modeled for the system without an operator present and also changed with different operators and possibly during testing with the same operator. In order to control for the existence of the uncertainty in the plant model due to the uncertainty in the gripping, a two degree-of-freedom controller structure was used. The feedback controller was designed to address the problem of robustness of the system associated with gripping and the native dynamics of the system and the feedforward controller was designed to improve the response of the simulator.

Another known difficulty with the simulator apparatus was the effect of the steering wheel and the steering column. The steering wheel added a coupling between the $\mathrm{z}$ - and y-axes while the steering column acted as a cantilever beam. Furthermore, the same simulator column and wheel assembly will being used to replicate the steering wheel and column assembly from an arbitrary automobile. In order to deal with the unknown plant variations, coupling between the axes and the associated beam resonances, a two input one output stabilizing feedback controller is synthesized using LMI techniques.

Using standard LMI techniques for a discrete system (Iwasaki, 1993, Skelton, et al., 1998), a MATLAB script was written to synthesize a discrete controller capable of making the closed-loop system quadraticly stable. Given the following discrete-time LTI plant model:

$$
\begin{aligned}
& \mathbf{x}(k+1)=\mathbf{A} \mathbf{x}(k)+\mathbf{B}_{u} \mathbf{u}(k)+\mathbf{B}_{w} \mathbf{w}(k) \\
& \mathbf{y}(k)=\mathbf{C}_{y} \mathbf{x}(k)+\mathbf{D}_{y u} \mathbf{u}(k) \\
& \mathbf{z}(k)=\mathbf{C}_{z} \mathbf{x}(k)+\mathbf{D}_{z u} \mathbf{u}(k)
\end{aligned} .
$$

Without loss of generality, let $\mathbf{D}_{\mathrm{yu}}=\mathbf{D}_{\mathrm{zu}}=0$. The input vector, $\mathbf{u}$, contains all controllable inputs while the input vector, w, contains all exogenous inputs. The output vector, $\mathbf{y}$, is the set of all measurable outputs needed for the control and the output vector, $\mathbf{z}$, is the set of all non-measurable outputs and those measurable outputs not needed for control. The system matrix $\mathbf{A}$ is defined to be of size $\mathrm{n} \times \mathrm{n}$. The input matrices $\mathbf{B}_{\mathrm{u}}$ and $\mathbf{B}_{\mathrm{w}}$ are defined to be of size $\mathrm{n} \times \mathrm{n}_{\mathrm{u}}$ and $\mathrm{n} \times \mathrm{n}_{\mathrm{w}}$, respectively. Similarly, the output matrices $\mathbf{C}_{\mathrm{y}}$ and $\mathbf{C}_{\mathrm{z}}$ are of size $n_{y} \times n$ and $n_{z} x n$, respectively. The values of the $\mathbf{A}, \mathbf{B}_{\mathrm{u}}, \mathbf{B}_{\mathrm{w}}, \mathbf{C}_{\mathrm{y}}$ and $\mathbf{C}_{\mathrm{z}}$ matrices were taken from the system model discussed in Section 2.

A full order discrete-time LTI controller of the form

$\mathbf{x}_{c}(k+1)=\mathbf{A}_{c} \mathbf{x}_{c}(k)+\mathbf{B}_{c} \mathbf{y}(k)$.

$\mathbf{u}(k)=\mathbf{C}_{c} \mathbf{x}_{c}(k)+\mathbf{D}_{c} \mathbf{y}(k)$

is to be computed. The closed-loop system matrix, $\mathbf{A}_{\mathrm{cl}}$, can be expressed as

$$
\begin{aligned}
& \mathbf{A}_{c l}=\left[\begin{array}{cc}
\mathbf{A}_{c}+\mathbf{B}_{u} \mathbf{D}_{c} \mathbf{C}_{y} & \mathbf{B}_{u} \mathbf{C}_{c} \\
\mathbf{B}_{c} \mathbf{C}_{y} & \mathbf{A}_{c}
\end{array}\right] \\
& A_{c l}=\left[\begin{array}{cc}
\mathbf{A} & \mathbf{0} \\
\mathbf{0} & \mathbf{0}
\end{array}\right]+\left[\begin{array}{cc}
\mathbf{0} & \mathbf{B}_{u} \\
\mathbf{I} & \mathbf{0}
\end{array}\right]\left[\begin{array}{cc}
\mathbf{A}_{c} & \mathbf{B}_{c} \\
\mathbf{C}_{c} & \mathbf{D}_{c}
\end{array}\right]\left[\begin{array}{cc}
\mathbf{0} & \mathbf{I} \\
\mathbf{C}_{y} & \mathbf{0}
\end{array}\right] \cdot \\
& A_{c l}=A_{0}+B_{0} \Omega C_{0}
\end{aligned}
$$

The condition for quadratic stability is

$\left[\begin{array}{c}\mathbf{x}(k) \\ \mathbf{x}_{c}(k)\end{array}\right]^{*}\left[\left(\mathbf{A}_{0}+\mathbf{B}_{0} \mathbf{\Omega} \mathbf{C}_{0}\right)^{*} \mathbf{P}\left(\mathbf{A}_{0}+\mathbf{B}_{0} \mathbf{\Omega} \mathbf{C}_{0}\right)-\mathbf{P}\right]\left[\begin{array}{c}\mathbf{x}(k) \\ \mathbf{x}_{c}(k)\end{array}\right]<0$

In order to formulate the above system into a convex optimization problem, it was necessary to apply the elimination lemma for discrete LMI conditions stated by Skelton, et al. (1998). 
If MATLAB is to be used to solve the LMI formulation, one needs to note the difference in the null space definition between MATLAB and that of the elimination lemma as stated by Skelton, et al. (1998). The MATLAB null command produces a null space described by

$$
\mathbf{A}^{\perp}=\operatorname{null}(\mathbf{A})
$$

$\mathbf{A A}^{\perp}=0$

In order to produce the desired null space for use in the elimination lemma, it is necessary to use the form:

$\mathbf{B}^{\perp}=\left[\operatorname{null}\left(\mathbf{B}^{\star}\right)\right]^{\star}$

$\mathbf{B}^{\perp} \mathbf{B}=0$

Another important issue with using the MATLAB LMI toolbox was the effect of rounding errors. During the modeling of the simulator it was found that higher order models, typically above 40 states, generated an excellent fit the experimental frequency response data. However, with more than 40 states in the plant model, the associated LMI solution was very sensitive to rounding errors and failed the quadratic stability condition stated by Eq. (4). It was necessary to generate a balanced realization of the system model before applying the LMI conditions.

\subsection{Path Shaping Feedforward Controller}

With the stabilizing controller in the feedback loop, a feedforward controller is designed to achieve the desired performance. As designed, the feedback controller was not designed to satisfy the specific performance condition. A feedforward controller for shaping the input was designed by inversion of the closed loop system designed above. One thing to note in the derivation of the feedforward controller was that the feedback controller was selected to be of relatively low order in order to facilitate real-time implementation. The feedforward controller was under no restrictions for size as it can be applied off-line in the actual implementation.

The stabilizing feedback controller together with the plant model combined to form the closed loop system

$G_{C L}=G_{s y s}\left(I+C_{s y s} G_{s y s}\right)^{-1}$.

The ideal feedforward controller is the inverse of the closed loop system $G_{C L}$. However, inversion of the closed loop system, $G_{C L}$, with one input and two outputs needs additional consideration. The closed loop system can be described as

$$
\left[\begin{array}{l}
z \\
y
\end{array}\right]=G_{C L} \cdot u_{z} \text {. }
$$

The pseudo-inverse of the $2 \times 1$ transfer matrix $G_{C L}$ can be computed by

$\left[G_{C L}{ }^{T} G_{C L}\right]^{-1}\left[G_{C L}{ }^{T}\right]\left[\begin{array}{l}z \\ y\end{array}\right]=u_{z}$.
$G_{C L}=\left[\begin{array}{l}G_{C L, z} \\ G_{C L, y}\end{array}\right]$.

This inverted closed loop system provides a relationship between the desired closed loop system outputs $z_{d}$ and $y_{d}$ and the system input $u_{z}$ required to achieve these outputs. This relationship can be rewritten as

$\frac{1}{{G_{C L, z}{ }^{2}+G_{C L, y}{ }^{2}}_{G^{2}}}\left[G_{C L, z}\left[\begin{array}{l}z_{d} \\ y_{d}\end{array}\right]=u_{c}\right.$.

Inserting Eqs. (10) and (11) into the original closed loop system in Eq. (8) gives

$\left.\left[\begin{array}{l}z \\ y\end{array}\right]=\left[\begin{array}{l}G_{C L, z} \\ G_{C L, y}\end{array}\right] \frac{1}{{G_{C L, z}{ }^{2}+G_{C L, y}{ }^{2}}_{G_{C L, z}}} G_{C L, y}\right]\left[\begin{array}{l}z_{d} \\ y_{d}\end{array}\right]$.

Simplifying this expression yields

$\left[\begin{array}{c}z \\ y\end{array}\right]=\frac{1}{{G_{C L, z}{ }^{2}+G_{C L, y}{ }^{2}}^{2}}\left[\begin{array}{cc}G_{C L, z}{ }^{2} & G_{C L, z} G_{C L, y} \\ G_{C L, y} G_{C L, z} & G_{C L, y}{ }^{2}\end{array}\right]\left[\begin{array}{l}z_{d} \\ y_{d}\end{array}\right]$. (13)

In addition to the complexity in inverting the closedloop system, the resulting pseudo-inverse is also noncausal for the strictly proper closed-loop system. If the closed-loop system has non-minimum phase zeros, then the pseudo-inverse will be unstable. To address these issues, the zero phase error tracking control concept was used.

The first step to achieve zero phase error is to partition the closed-loop transfer function into transfer functions for the z-axis and the $y$-axis and expressed as

$G_{C L}\left(z^{-1}\right)=\left[\frac{z^{-d z} B_{Z}\left(z^{-1}\right)}{A_{Z}\left(z^{-1}\right)}, \frac{z^{-d y} B_{Y}\left(z^{-1}\right)}{A_{Y}\left(z^{-1}\right)}\right]$,

where $d z$ and $d y$ are the relative degrees of the closedloop transfer functions for the $\mathrm{z}$ - and $\mathrm{y}$-axis, respectively. Note that $B_{Z}\left(z^{-1}\right)$ and $B_{Y}\left(z^{-1}\right)$ contain both the minimum phase and non-minimum phase zeros of the closed loop system. The non-minimum phase zeros caused the instability mentioned above. To address this, first partition $B_{Z}\left(z^{-1}\right)$ and $B_{Y}\left(z^{-1}\right)$ into the stable and unstable portions,

$$
\begin{gathered}
B_{Z}\left(z^{-1}\right)=B_{Z}{ }^{+}\left(z^{-1}\right) B_{Z}{ }^{-}\left(z^{-1}\right) \quad \text { and }, \\
B_{Y}\left(z^{-1}\right)=B_{Y}{ }^{+}\left(z^{-1}\right) B_{Y}{ }^{-}\left(z^{-1}\right)
\end{gathered}
$$

where the stable roots are collected into $B_{Z}{ }^{+}\left(z^{-1}\right)$ and $B_{Y}{ }^{+}\left(z^{-1}\right)$, respectively. The unstable roots are collected into the terms $B_{Z}^{-}\left(z^{-1}\right)$ and $B_{Y}^{-}\left(z^{-1}\right)$, respectively. With the partitioned polynomials, the individual transfer functions can be inverted and written as

$C_{F F, i}=\left[\frac{z^{d z} A_{Z}\left(z^{-1}\right)}{B_{Z}^{+}\left(z^{-1}\right) B_{Z}^{-}\left(z^{-1}\right)}, \frac{z^{d y} A_{Y}\left(z^{-1}\right)}{B_{Y}^{+}\left(z^{-1}\right) B_{Y}^{-}\left(z^{-1}\right)}\right]$.

Since one cannot perfectly cancel the unstable (nonminimum phase) zeros of the closed loop transfer function, for tracking purpose, it would be desirable to compensate for the phase lag of the strictly proper closed-loop system. Since $\angle\left[N(z) \cdot N\left(z^{-1}\right)\right]=0$ for $z \in C$, the following feedforward controller

Note that if $G_{C L}$ is written as 
$C_{F F}=\left[\frac{z^{d z} A_{Z}\left(z^{-1}\right) B_{Z}^{-}(z)}{B_{Z}{ }^{-}\left(z^{-1}\right)\left[B_{Z}{ }^{-}(1)\right]^{2}}, \frac{z^{d y} A_{Y}\left(z^{-1}\right) B_{Y}^{-}(z)}{B_{Y}{ }^{+}\left(z^{-1}\right)\left[B_{Y}{ }^{-}(1)\right]^{2}}\right]$,

together with the closed-loop system $\mathrm{G}_{\mathrm{CL}}$ will result in a input/output system with zero phase lag. The scalar terms in the denominators, $\left[B_{Z}{ }^{-}(1)\right]^{2}$ and $\left[B_{Y}{ }^{-}(1)\right]^{2}$, are added to ensure a unity steady state gain.

As in the stabilizing feedback controller design, rounding errors were particularly significant in this design. Unlike the feedback controller, no effort was made to limit the size of the plant model to match the computational limits of the feedforward controller design. Although these results were derived using transfer functions, in MATLAB it was far more accurate to express the intermediate steps as zero-polegain structures. This limited the rounding errors to an acceptable level.

The implementation of the filter was not a trivial task. Converting the zero-pole-gain structure to a transfer function in order to take advantage of the filtering functions built into MATLAB again introduced significant rounding errors. Since the reference signals for testing were generated by a finite sum of sinusoids, it was possible to apply the magnitude and phase of the filter to each sinusoid. In addition, each sinusoid was scaled by the inverse of the magnitude of the simulated combination of feedforward and feedback control of the system model. In this manner, both the magnitude and phase errors were eliminated for all simple reference signals.

\section{RESULTS}

To verify theoretical controller performance, a simulation study is performed. The reference was selected as a $30-\mathrm{Hz}$ sinusoidal signal. This frequency was chosen to avoid resonances in the plant model.

Figure 3 shows a simulated $50-\mathrm{Hz}$ reference signal, the output of the feedforward controller and the simulated response of the system in the z-axis to the $50-\mathrm{Hz}$ reference input with appropriate magnitude calibration in the preprocessing. As expected from the full system simulated frequency response, there is minimal phase and magnitude error. Figure 4 shows the percent error between the simulated response of the system in the zaxis and the peak simulated reference signal. This simulated result could easily be extended to any signals comprised of a finite sum of sinusoids.

Some post processing was necessary in order to present experimental results. No signal was available to trigger the collection of data. Instead, data collection was triggered manually, and the experimental feedforward filtered signal was compared to the simulated feedforward signal. The actual reference signal was not recorded. By minimizing the sum of the squared error between the simulated and experimental feedforward signals, it was possible to align the experimental data to the simulated reference signal used.

Figure 5 shows the simulated $\mathrm{z}$-axis reference signal with experimental z-axis acceleration. A slight phase error can be seen between the reference and output. The magnitude error is minimal. Figure 6 shows the percentage error between the experimental output and the peak simulated reference signal.

\section{CONCLUSIONS}

The goals of this project were to build a simulator capable of reproducing an arbitrary vibration signal between $5-\mathrm{Hz}$ and $250-\mathrm{Hz}$ and to control the apparatus in order to increase tracking performance during the reproduction of an arbitrary vibration signal. An apparatus was built that was capable of reproducing a vibration input between $5-\mathrm{Hz}$ and $250-\mathrm{Hz}$ and controlled to increase tracking performance. In simulation and experimental testing, the system proved capable to reproducing signals with minimal phase error and large magnitude error. For reference signals comprised of sums of sinusoidal signals, pre-scaling of the reference signal was used to achieve minimal magnitude error. The two degree-of-freedom controller was successful in the goal of eliminating phase error between reference input and experimental output while being robust to variations in occupant grip.

\section{REFERENCES}

Akaike, H. (1972). "Information theory and extension of the maximum likelyhood principle", Proceedings of the $2^{\text {nd }}$ International Symposium on Information Theory, Supplement to Problems of Control and Information Theory, pp. 267-281.

Akaike, H. (1974). "A new look at the statistical model identification", IEEE Transactions on Automatic Control, Vol. AC-19, No. 6, pp. 716-723.

Balakrishnan, V., Boyd, S., Feron E., and El Ghaoui, L.. (1994). Linear Matrix Inequalities in System and Control Theory, SIAM: Philadelphia.

Bolanowski, S.J., Gescheider, G.A., Verrillo, R.T., and Checkoky, C.M. (1988). "Four channels mediate the mechanical aspects of touch", Journal of the Acoustical Society of America, Vol. 82, No. 5, pp. 1680-1694.

Brisben, A.J., Hsiao, S.S., and Johnson, K.O. (1999). "Detection of vibration transmitted through an object grasped in the hand", The American Physiological Society, pp. 1548-1558.

Gescheider, G.A., O’Malley, M.J., and Verrillo, R.T. (1983). "Vibrotactile forward masking: evidence for channel independence", Journal of the Acoustical Society of America, Vol. 74, No. 2, pp. 474-485.

Skelton, R.E., Iwasaki, T. and Grigoriadis, K. (1998). A Unified Algebraic Approach to Linear Control Design, Taylor and Francis: London, 1998.

Iwasaki, T. (1993). A Unified Matrix Inequality Approach to Linear Control Design, Ph.D. 
Dissertation, Purdue University: West Lafayette, Indiana, 1993.

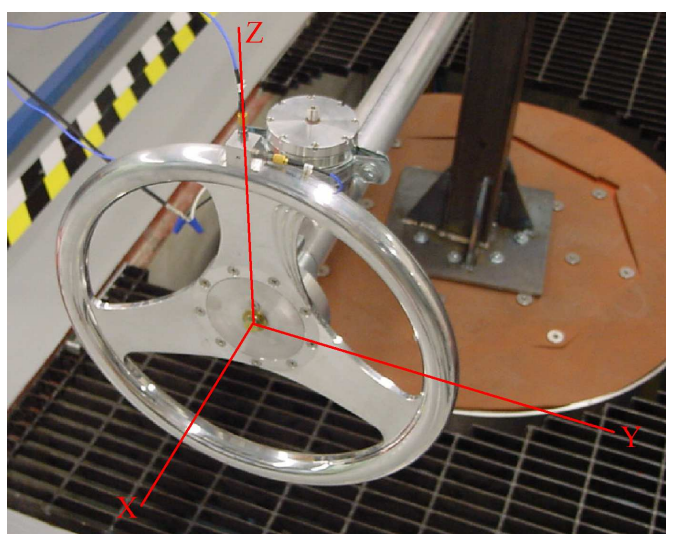

Fig. 1. Photograph of assembled steering wheel and column with coordinate system.
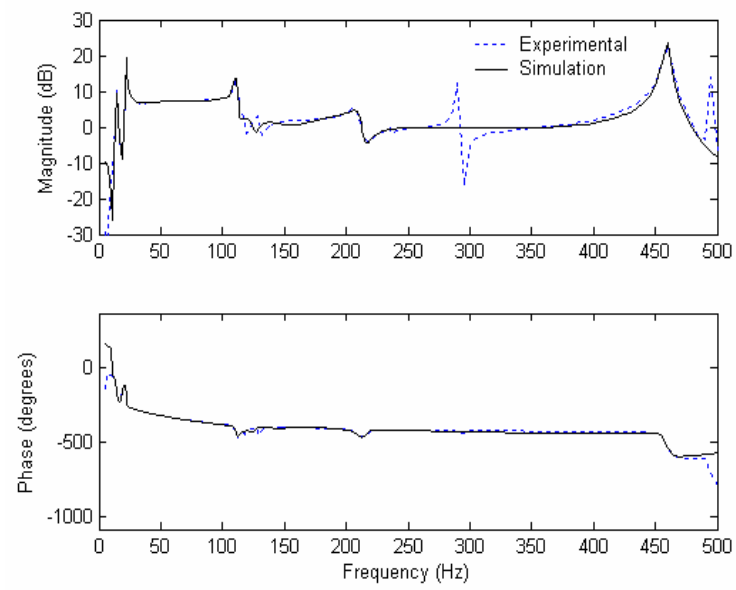

Fig. 2. Open-loop z-axis frequency response of apparatus (dotted line) and $24^{\text {th }}$ order plant model (solid line).
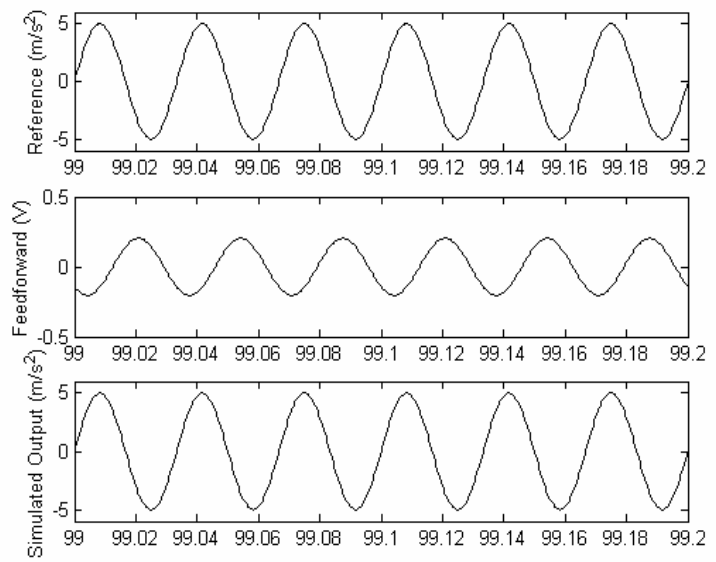

Fig. 3. Simulated reference signal at $30-\mathrm{Hz}$ in $\mathrm{z}$-axis (top) with simulated response of feedforward controller (middle) and simulated z-axis response of $24^{\text {th }}$ order plant model with two degree-offreedom controller (bottom).

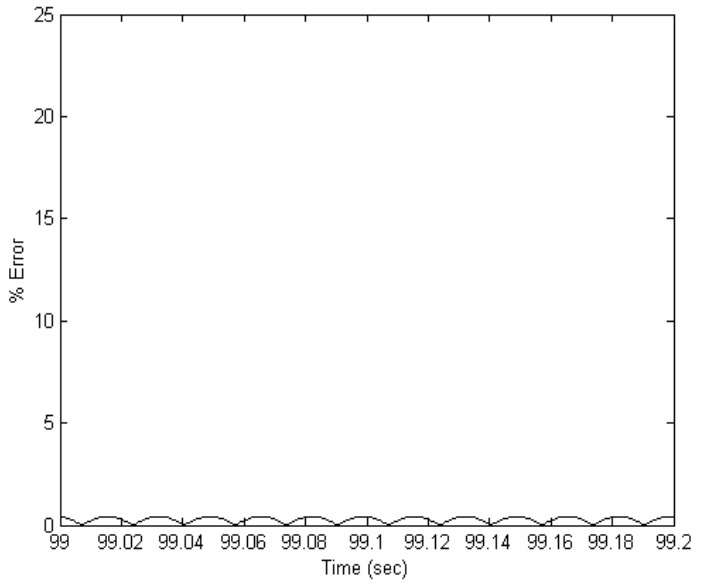

Fig. 4. Percentage error between simulated response of $24^{\text {th }}$ order plant model with two degree-offreedom controller and peak simulated reference input described by Fig. 3.

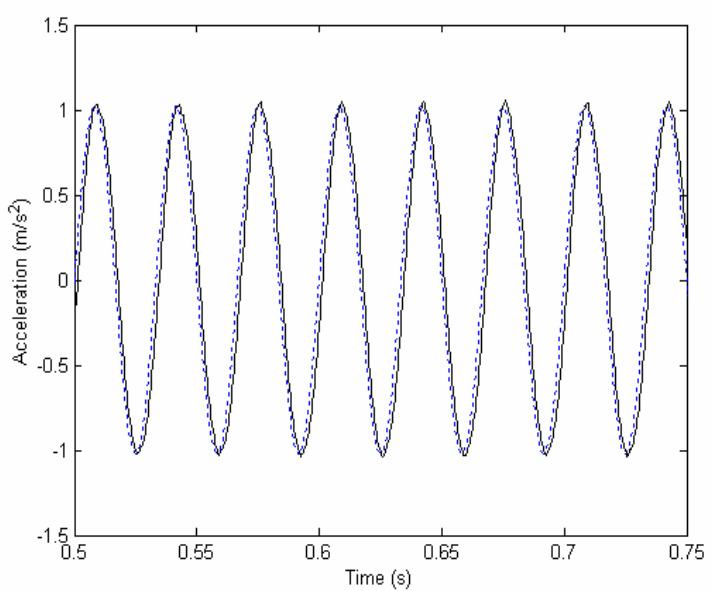

Fig. 5. Simulated reference signal at 30-Hz (dotted line) and experimental output to the reference (solid line).

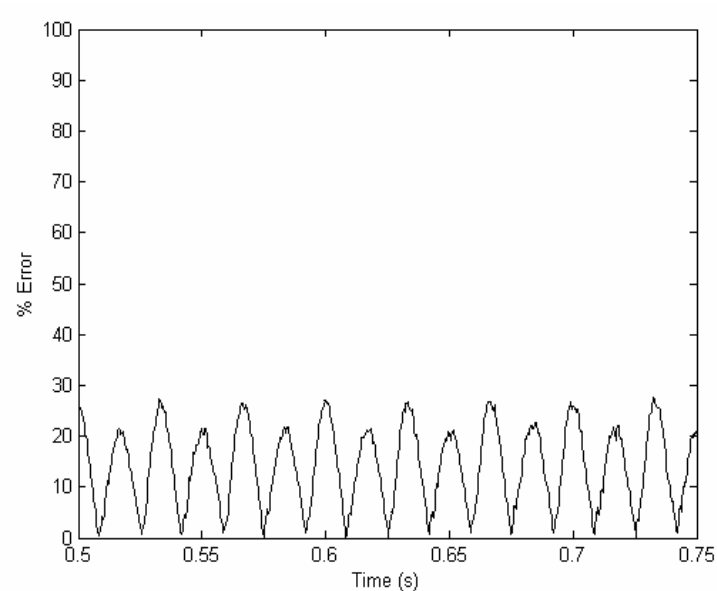

Fig. 6. Percentage error between experimental output and simulated peak reference input, as shown in Fig. 5. 